\title{
Coffee Consumption and Risk of Prostate Cancer in Local, Advanced, and Fatal Grades: A Meta-Epidemiological Study of Prospective Cohort Studies
}

\author{
Jong-Myon Bae \\ Department of Preventive Medicine, Jeju National University School of Medicine, Jeju, Korea
}

\begin{abstract}
There were conflict reports among the previous systematic reviews conducted to evaluate the association between coffee consumption and risk of prostate cancer. This meta-epidemiological study aimed to evaluate the reasons for conflicting findings among previous studies and then estimate a more valid summary effect size of the association between coffee consumption and prostate cancer risk in grades and all cases. The selection criteria were defined that a prospective cohort study conducted to evaluate coffee consumption and risk of prostate cancer incidence with reporting adjusted relative risk (RR) and its $95 \%$ confidence interval $(95 \% \mathrm{Cl})$. Random effect model meta-analysis and 2-stage fixed-effect dose-response meta-analysis were performed by cancer grades. From a total of 12 prospective cohort studies were selected, 11 all, 4 local, 5 advanced, and 4 fatal cohorts were organized. There was an inverse association between coffee consumption and the risk of prostate cancer in all cases with statistical significance (summary RR, $0.91 ; 95 \% \mathrm{Cl}, 0.84-0.98$; I-squared $=49.1 \%$ ). Dose-response meta-analysis showed that the unit's decreasing risk levels were $1.0 \%$ in all cases, $1.1 \%$ in localized cases, and $2.5 \%$ in fatal cases, respectively. There is an inverse association between coffee consumption and risk of prostate cancer in local and fatal grades, not in advanced grade. And the main reasons for conflict results among the previous studies would be due to noncomprehensive searching strategies, the interdifferent definition of selection criteria, inaccurate extraction of information, and inadequate adjustment for potential confounders. (Korean J Urol Oncol 2021;19:16-22)
\end{abstract}

Key Words: Coffee $\cdot$ Prostate neoplasm - Systematic review $\cdot$ Meta-analysis

\section{INTRODUCTION}

Prostate cancer ranks 'the second most frequent cancer and the fifth leading cause of cancer death in men' in

Received November 5, 2020, Revised December 1, 2020,

Accepted December 10, 2020

Corresponding Author: Jong-Myon Bae

Department of Preventive Medicine, Jeju National University

School of Medicine, 102 Jejudaehak-ro, Jeju 63243, Korea

Email: jmbae@jejunu.ac.kr

Tel: +82-64-755-5567, Fax: +82-64-725-2593

ORCID: https://orcid.org/0000-0003-3080-7852
GLOBOCAN 2018. ${ }^{1}$ While age and genetic factors are the well-known risk factors, some modifiable risk factors as westernized diet, obesity, and physical inactivity are positively associated with prostate cancer. ${ }^{2}$ Since Stocks $^{3}$ reported the positive correlation between coffee consumption and prostate cancer mortality through an ecological study in 1970, several epidemiological studies have been conducted to investigate the association between coffee consumption and risk of prostate cancer. ${ }^{4}$ And experimental studies have reported that several compounds of coffee could alter cancer risk through inhibiting DNA methylation, stimulating antioxidant defense mechanisms, or increasing

This is an Open Access article distributed under the terms of the Creative Commons Attribution Non-Commercial License (http://creativecommons.org/licenses/by-nc/4.0/) which permits unrestricted non-commercial use, distribution, and reproduction in any medium, provided the original work is properly cited. 2021 (C) Copyright The Korean Urological Oncology Society and The Korean Prostate Society. All Rights Reserved. 
insulin sensitivity, etc. ${ }^{5}$

Table 1 shows the results of systematic reviews (SRs) conducted to investigate the hypothesis. With the exception of Park et al., ${ }^{5}$ which was performed for the first time, and Xia et al., ${ }^{6}$ which was performed last, the other 7 SRs of population-based cohort studies ${ }^{7-13}$ were reported to significantly reduce the risk of prostate cancer in all cases from coffee consumption. In other words, statistical significance was continuously reported in the 7 SRs published in 20112016, but statistical significance disappeared in Xia et al. ${ }^{6}$ published in 2017. Especially, the selected number of cohort studies in 2 recent $\mathrm{SR}^{6,13}$ was the same, but the results were contrary. And I-squared value indicating heterogeneity level also varied from $30.3 \%$ to $64.8 \%$.

Therefore, this meta-epidemiological study ${ }^{14}$ aimed to evaluate the reasons for conflicting findings among SRs and then estimate a more valid summary effect size of the association between coffee consumption and prostate cancer risk in local, advanced, fatal grade, as well as all cases.

\section{MATERIALS AND METHODS}

To estimate more valid results, the stricter selection criteria were applied such as a prospective cohort study conducted to evaluate coffee consumption and risk of prostate cancer incidence with reporting adjusted relative risk (aRR) and its $95 \%$ confidence interval (CI). Thus, 10 of 16 studies selected by SRs in Table 1 were met the selection criteria. $^{15-24}$

Considering that the most recent publication year among them was in $2015,{ }^{24}$ it was necessary to secure cohort studies that met the selection criteria among the papers published until the 10 October 2020. To do this, the 'cited by' option for 10 studies $^{15-24}$ and the 'similar articles' of Xia et al. $^{6}$ were applied using the citation discovery tool provided by PubMed. ${ }^{25}$ If the selected articles were duplicated in cohort participants, the article reporting more information for meta-analysis was selected. Cohorts from final selected studies were arranged by cancer grades such as localized,

Table 1. Results of previous meta-analysis of population-based cohort studies

\begin{tabular}{|c|c|c|c|c|c|}
\hline Study & Searching & Cancer grade & Selected cohort studies & sRR $(95 \% \mathrm{CI})$ & I-squared $(\%)$ \\
\hline Park et al. ${ }^{5}$ (2010) & Aug 2009 & All & 4 & $1.06(0.83-1.35)$ & 0.0 \\
\hline Yu et al. ${ }^{7}$ (2011) & Mar 2010 & All & 5 & $0.79(0.61-0.98)$ & 57.1 \\
\hline \multirow[t]{4}{*}{ Lu et al. $^{8}$ (2014) } & Jun 2013 & All & 9 & $0.89(0.84-0.95)$ & 8.0 \\
\hline & & Localized & & $0.89(0.83-0.96)$ & 0.0 \\
\hline & & Advanced & & $0.85(0.58-1.12)$ & 68.9 \\
\hline & & Fatal & & $0.64(0.47-0.80)$ & 47.8 \\
\hline Cao et al. ${ }^{9}$ (2014) & Jun 2013 & All & 10 & $0.88(0.82-0.95)$ & 31.9 \\
\hline \multirow[t]{4}{*}{ Zhong et al. ${ }^{10}$ (2014) } & Jul 2013 & All & 12 & $0.83(0.72-0.96)$ & 66.5 \\
\hline & & Local & & $0.74(0.47-1.17)$ & 79.4 \\
\hline & & Advanced & & $0.71(0.47-1.08)$ & 75.8 \\
\hline & & Fatal & & $0.61(0.42-0.90)$ & 61.4 \\
\hline \multirow[t]{4}{*}{ Huang et al. ${ }^{11}$ (2014) } & Aug 2013 & All & 8 & $0.86(0.79-0.95)$ & 25.0 \\
\hline & & Local & & $0.90(0.83-0.97)$ & 0.0 \\
\hline & & Advanced & & $0.73(0.50-1.07)$ & 72.8 \\
\hline & & Fatal & & $0.70(0.46-1.05)$ & 57.5 \\
\hline \multirow[t]{4}{*}{ Liu et al. ${ }^{12}$ (2015) } & Aug 2013 & All & 13 & $0.90(0.85-0.95)$ & 17.5 \\
\hline & & Local & 5 & $0.89(0.83-0.96)$ & 0.0 \\
\hline & & Advanced & 6 & $0.82(0.61-1.10)$ & 64.1 \\
\hline & & Fatal & 4 & $0.76(0.55-1.06)$ & 49.1 \\
\hline Wang et al. ${ }^{13}$ (2016) & Jul 2015 & All & 14 & $0.89(0.84-0.93)$ & 30.3 \\
\hline \multirow[t]{3}{*}{ Xia et al. ${ }^{6}(2017)$} & Jul 2016 & All & 14 & $0.97(0.84-1.12)$ & 64.8 \\
\hline & & Local & 4 & $0.90(0.83-0.97)$ & 0.0 \\
\hline & & Advanced & 4 & $0.84(0.58-1.21)$ & 72.0 \\
\hline
\end{tabular}

SRR: summary relative risk, CI: confidence intervals. 
advanced, and fatal. Based on the tumor-node-metastasis staging score suggested by 3 studies, ${ }^{19,21,22}$ the localized group was defined as 'T1-2 and NX-0 and MX-0.' And the advanced group was defined as 'T3-4, N1 or M1.'

As the lowest level was defined as 'never or seldom' categories, ${ }^{9}$ aRR and its $95 \%$ CI of the highest category in each study were extracted. To evaluate potential effects of confounders, the author checked whether alcohol habits, body mass index, or physical activity were adjusted in estimating RR in each study and appraised the level of smoking status adjustment. The high level was defined as adjusting for the intensity as well as the status, while the low level was defined as adjusting for the only status or none.

The heterogeneity level of the cohorts was evaluated by I-squared value $(\%)$. In the case of over $50 \%$, the random effect model meta-analysis of highest versus lowest method (HLMA) was performed. Egger test as well as funnel plot were performed to evaluate the publication bias. Subgroup analyses by adjustment of 4 potential confounders - alcohol habit, body mass index, physical activity, and level of smoking status-were conducted.

A 2-stage fixed-effect dose-response meta-analysis (DRMA) after checking p-value of Goodness-of-fit over 0.10 was performed to calculate the risk of prostate cancer per unit dose of coffee consumption (cup per day). ${ }^{26}$ The author defined $250 \mathrm{~g}$ of coffee equal to 1 cup. ${ }^{10}$ Using a restricted cubic spline model with 4 knots at fixed percentiles $(5 \%$, $35 \%, 65 \%$, and $95 \%$ ) of the distribution, the linear relationship was confirmed based on the coefficients of the second and third spline. The level of statistical significance was set at 0.05 .

\section{RESULTS}

As of 10 October 2020, a total of 247 papers cited 10 articles $^{15-24}$ and 104 papers were similar to Xia et al. ${ }^{6}$ through the citation discovery tool provided by PubMed. The author secured newly 3 studies that met the selection criteria from them. ${ }^{27-29}$ While Wilson et al. ${ }^{19}$ and Russnes et al. $^{27}$ have the same cohort participants, the author selected Wilson et al. ${ }^{19}$ because it had more information for HLMA and DRMA. Thus, a total of 12 prospective cohort studies were selected for HLMA, finally (Fig. 1). ${ }^{15-24,28,29}$ A total of 920,547 persons participated and 53,943 cases oc- curred during the follow-up periods. There were 5 in North America, 5 in Europe, and 2 in Asia according to regional distribution. And 11 all, 4 local, 5 advanced, and 4 fatal cohorts from 12 studies were organized.

Fig. 2 shows the extraction values from each study and their HLMA results by cancer grades. The risk of prostate cancer in localized, advanced, and fatal grade according to coffee consumption did not show the statistical significance. But there was an inverse association between coffee consumption and the risk prostate cancer in all cases with statistical significance (summary RR [sRR], 0.91; 95\% CI, 0.840.98 ; I-squared $=49.1 \%)$. The $p$-value of Egger test $(p=0.435)$ and funnel plot (Fig. 3) did not show a publication bias.

Table 2 illustrates the results of subgroup analysis to find out the influence of 4 potential confounders in cohorts of all cases showing statistical significance in Fig. 2. Statistical significance maintained when the level of adjusting smoking status was high, and alcohol habits, obesity, or physical activity were adjusted.

Two of 12 studies had not the information needed to conduct a DRMA. ${ }^{16,28}$ Table 3 summarizes the results of DRMA by cancer grades as well as of previous DRMA studies. Except for advanced cases, the risk of prostate cancer had the linearly inverse association by a cup of coffee consumed daily with statistical significance (Fig. 4). The unit's decreasing risk levels were $1.0 \%$ in all cases, $1.1 \%$ in localized cases, and $2.5 \%$ in fatal cases, respectively.

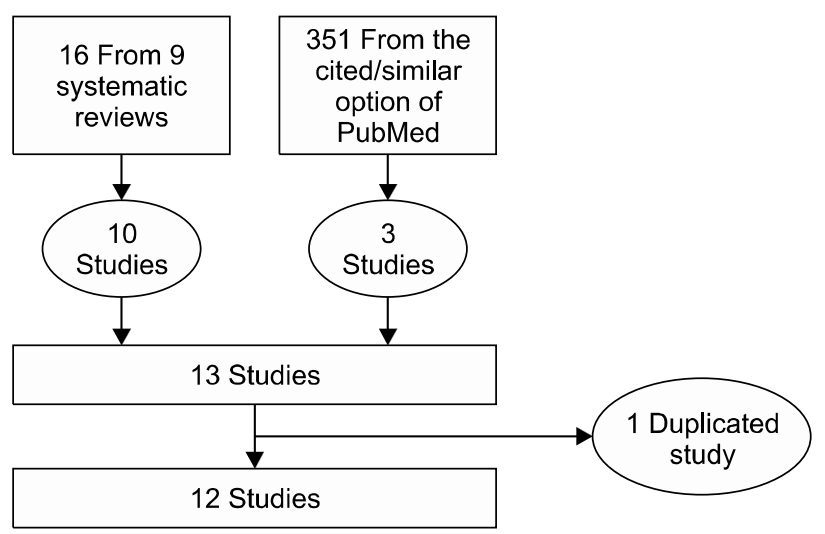

Fig. 1. Flow chart of the final selection of prospective cohort studies. 


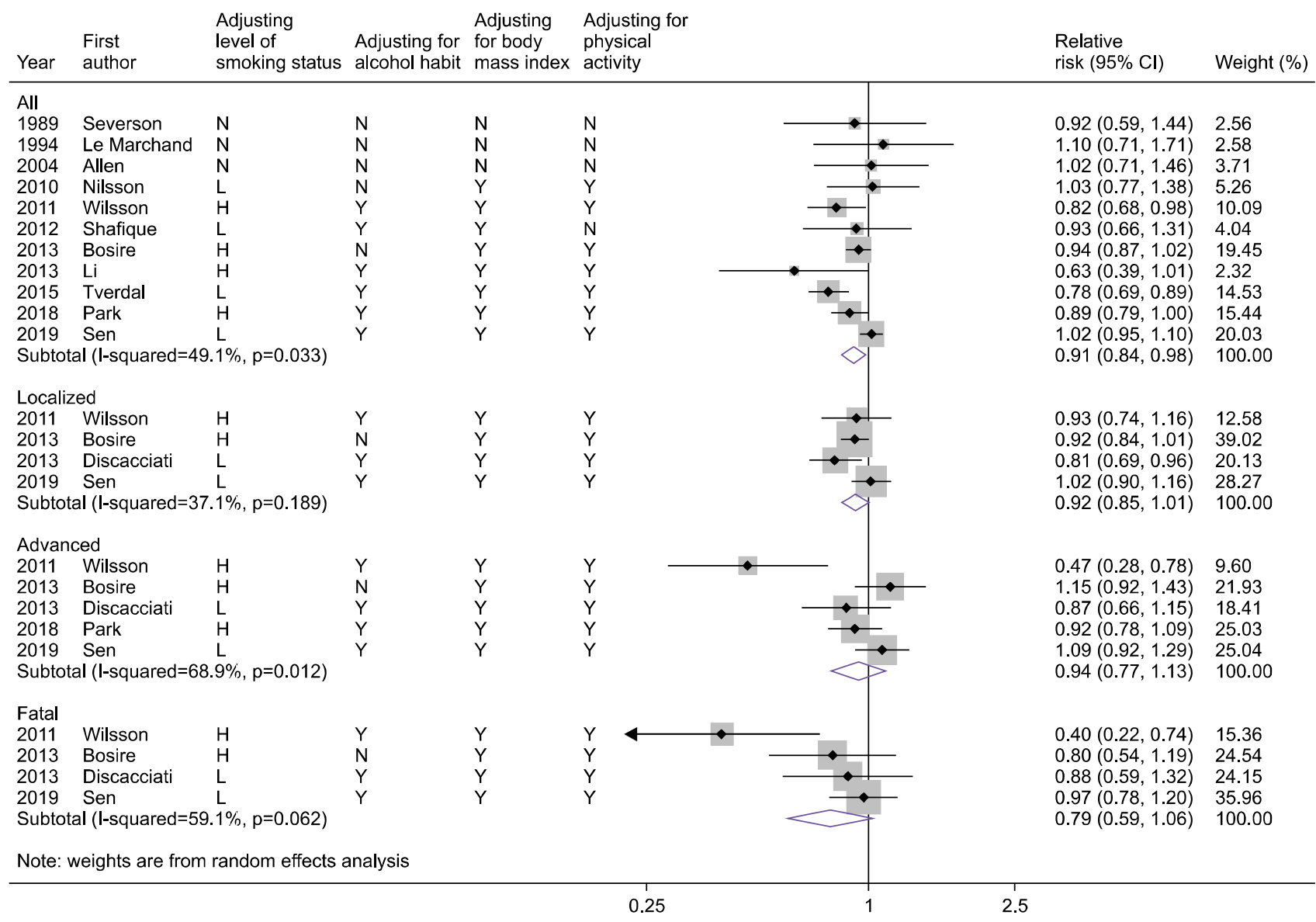

Fig. 2. Forest plot of meta-analysis using highest versus lowest method by prostate cancer grades. H: high, L: low, N: none, Y: yes, CI: confidence interval.

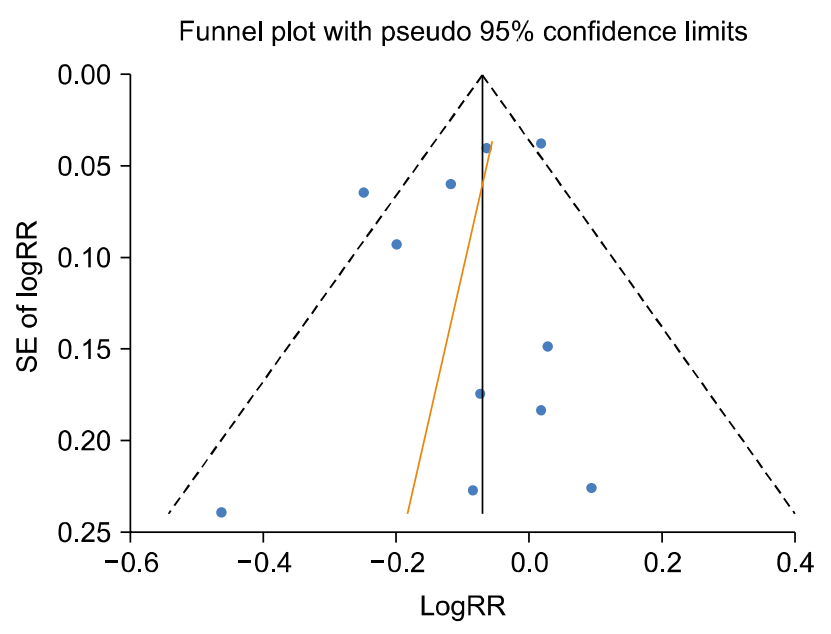

Fig. 3. Funnel plot. SE: standard error.

\section{DISCUSSION}

The HLMA showed that habitual coffee consumption would decrease prostate cancer risk in all cases (sRR, 0.91;
95\% CI, 0.84-0.98). Also, the DRMA suggested that habitual coffee consumption of a cup daily would decrease the risk in all cases of prostate cancer at $1.0 \%$. These findings suggested that the conflict reports among the previous SRs would be due to 3 main factors, such as noncomprehensive searching strategies, the interdifferent definition of selection criteria, and inaccurate extraction of information.

The reasons were as follows. Firstly, this meta-epidemiological study could secure 3 new cohort studies ${ }^{27-29}$ not included in the previous SRs introduced in Table 1. Considering that the most recent searching year in SRs was in 2016, ${ }^{6}$ Russnes et al. ${ }^{27}$ published in 2014 had to be evaluated. It is highly recommended to appraise and select the list of articles selected by the SRs published previously under the same research hypothesis. Secondly, there were 16 cohort studies selected by the previous 9 SRs, but 6 did not meet this study's selection criteria. It needs to apply stricter selection criteria to estimate a more valid effect size. 
Lastly, the author found some different RRs and their 95\% CI between forest plots in SRs and original articles. It is necessary to check the sameness of extracted values between the original article and the forest plot in order to prevent an error in the process of calcul ating $\log R R$ and SElogRR.

The subgroup analysis results in Table 2 could be interpreted that statistical significance disappeared when the level of adjusting smoking status was low, and alcohol hab-

Table 2. Subgroup analyses by potential confounders

\begin{tabular}{lc}
\hline \multicolumn{1}{c}{ Adjustment } & $\begin{array}{c}\text { Summary RR }(95 \% \mathrm{CI}) \\
\text { in cohorts of all cases }\end{array}$ \\
\hline $\begin{array}{l}\text { Level of smoking status } \\
\text { Low }\end{array}$ & $0.994(0.830-1.075)$ \\
High & $0.911(0.845-0.982)$ \\
Alcohol habit & \\
No & $0.952(0.885-1.024)$ \\
Yes & $0.870(0.768-0.984)$ \\
Body mass index & \\
$\quad$ No & $1.012(0.799-1.283)$ \\
Yes & $0.899(0.826-0.980)$ \\
Physical activity & \\
No & $0.985(0.810-1.197)$ \\
Yes & $0.897(0.819-0.982)$ \\
\hline
\end{tabular}

$\mathrm{RR}$ : relative risk, CI: confidence interval. its, obesity, or physical activity were not adjusted. These results are interesting because aRR moves toward a null, and statistical significance disappears as the intensity of adjustment increases in general. Based on these findings, it could be inferred that the interdifferent intensity of adjustment in selected studies could also cause conflicting results of SRs.

In contrast to HLMA in all cases, sRRs by cancer grades showed that habitual coffee consumption did not associate with prostate cancer risk in localized, advanced, and fatal

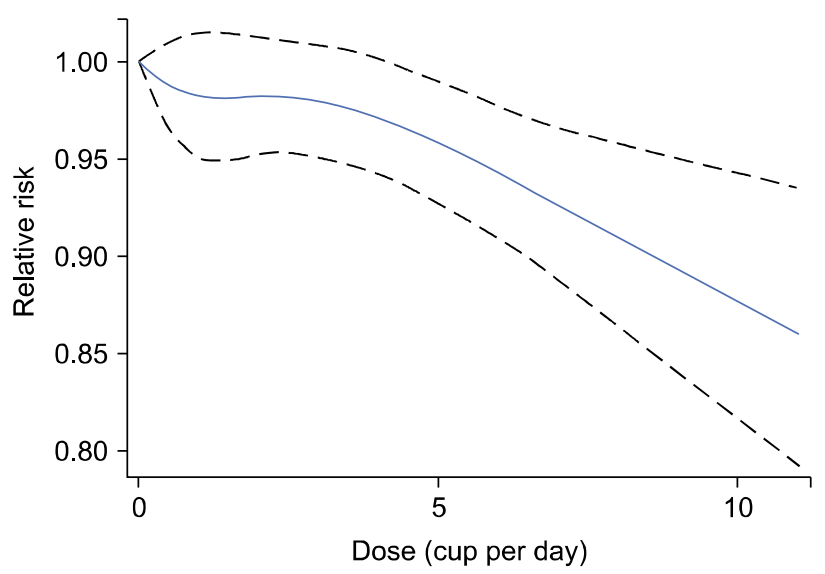

Fig. 4. Dose-response meta-analysis in 9 all cohorts per unit (cup daily).

Table 3. Results of dose-response meta-analysis

\begin{tabular}{|c|c|c|c|c|c|}
\hline Study & Unit (cup/day) & Cancer grade & $\begin{array}{l}\text { Selected cohort } \\
\text { studies }\end{array}$ & $\begin{array}{l}\text { p-value of } \\
\text { nonlinearity }\end{array}$ & $\mathrm{RR}(95 \% \mathrm{CI})$ \\
\hline \multirow[t]{4}{*}{ This study } & 1 & All & 9 & 0.32 & $0.990(0.985-0.996)$ \\
\hline & & Localized & 4 & 0.32 & $0.989(0.981-0.997)$ \\
\hline & & Advanced & 4 & 0.08 & $0.988(0.956-1.022)$ \\
\hline & & Fatal & 4 & 0.68 & $0.975(0.951-0.999)$ \\
\hline \multirow[t]{3}{*}{ Xia et al. $^{6}$ (2017) } & 1 & All & 10 & - & $0.990(0.980-1.000)$ \\
\hline & & Localized & 4 & - & $0.990(0.980-0.990)$ \\
\hline & & Advanced & 4 & - & $0.970(0.910-1.020)$ \\
\hline Wang et al. $^{13}$ (2016) & 2 & All & 10 & 0.15 & $0.970(0.960-0.980)$ \\
\hline Liu et al. ${ }^{12}$ (2015) & 2 & All & 9 & - & $0.975(0.957-0.995)$ \\
\hline Zhong et al. ${ }^{10}$ (2014) & 2 & All & 10 & 0.07 & $0.930(0.880-0.990)$ \\
\hline \multirow[t]{4}{*}{ Huang et al. ${ }^{11}$ (2014) } & 2 & All & 8 & 0.36 & $0.980(0.930-1.030)$ \\
\hline & & Localized & 4 & 0.47 & $0.980(0.920-1.040)$ \\
\hline & & Advanced & 5 & 0.74 & $0.980(0.860-1.120)$ \\
\hline & & Fatal & 3 & 0.62 & $0.950(0.770-1.160)$ \\
\hline \multirow[t]{3}{*}{ Discacciati et al. ${ }^{30}$ (2014) } & 3 & Localized & 4 & 0.12 & $0.970(0.950-0.990)$ \\
\hline & & Advanced & 4 & 0.18 & $0.910(0.800-1.050)$ \\
\hline & & Fatal & 3 & 0.36 & $0.890(0.820-0.970)$ \\
\hline
\end{tabular}

RR: relative risk, CI: confidence interval. 
cases (Fig. 2). Compared with the results presented by 3 grades in previous SRs in Table 1, there was no consistency. These results might be attributed to the decrease in the number of cohorts selected for meta-analysis as studies were divided by grades.

In contrast to HLMA in 3 grades, DRMA shows a linearly inverse association between habitual consumption of coffee and risk of prostate cancer in localized and fatal, except advanced grade. And the unit's decreasing risk level was higher in fatal grade rather than local grade. These results of this study were also confirmed in Xia et al. ${ }^{6}$ and Discacciati et al. ${ }^{30}$ in Table 3 . The different results between HLMA and DRMA might be caused by that DRMA uses the presented information relatively more than HLMA. If so, we could suggest that coffee consumption lowers the risk of prostate cancer in local and fatal grades based on DRMA results. On the other hand, the advanced grade had no statistical significance in DRMA compared to local and fatal grade. This could be interpreted as the fact that local and fatal showed a linear relationship $(\mathrm{p}>0.10)$, but advanced showed a nonlinear $(\mathrm{p}<0.10)$.

The advantages of this study are as follows. First, 3 additional cohort studies that were not selected in the existing SRs were additionally evaluated. Second, stricter selection criteria were applied, and then HLMA and DRMA were performed for 3 kinds of grade. Last, accordingly, the reasons for the conflict results among the existing SRs could be identified. On the other hand, the main limitation was that the effect on low and moderate dosage could not be analyzed as the category of coffee dosage was different for each study. Accordingly, DRMA was performed to confirm the level of risk reduction per unit consumption.

\section{CONCLUSIONS}

There is an inverse association between coffee consumption and risk of prostate cancer in local and fatal grades as well as all cases. And the main reasons of conflict results among the previous SRs would be due to noncomprehensive searching strategies, the interdifferent definition of selection criteria, inaccurate extraction of information, and inadequate adjustment for potential confounders.

\section{CONFLICT OF INTEREST}

The authors report no conflict of interest.

\section{REFERENCES}

1. Bray F, Ferlay J, Soerjomataram I, Siegel RL, Torre LA, Jemal A. Global cancer statistics 2018: GLOBOCAN estimates of incidence and mortality worldwide for 36 cancers in 185 countries. CA Cancer J Clin 2018;68:394-424.

2. Rawla P. Epidemiology of prostate cancer. World J Oncol 2019;10:63-89.

3. Stocks P. Cancer mortality in relation to national consumption of cigarettes, solid fuel, tea and coffee. $\mathrm{Br} \mathrm{J}$ Cancer 1970;24:215-25.

4. Arab L. Epidemiologic evidence on coffee and cancer. Nutr Cancer 2010;62:271-83.

5. Park CH, Myung SK, Kim TY, Seo HG, Jeon YJ, Kim $\mathrm{Y}$, et al. Coffee consumption and risk of prostate cancer: a meta-analysis of epidemiological studies. BJU Int 2010; 106:762-9.

6. Xia J, Chen J, Xue JX, Yang J, Wang ZJ. An up-to-date meta-analysis of coffee consumption and risk of prostate cancer. Urol J 2017;14:4079-88.

7. Yu X, Bao Z, Zou J, Dong J. Coffee consumption and risk of cancers: a meta-analysis of cohort studies. BMC Cancer 2011;11:96.

8. Lu Y, Zhai L, Zeng J, Peng Q, Wang J, Deng Y, et al. Coffee consumption and prostate cancer risk: an updated meta-analysis. Cancer Causes Control 2014;25:591-604.

9. Cao S, Liu L, Yin X, Wang Y, Liu J, Lu Z. Coffee consumption and risk of prostate cancer: a meta-analysis of prospective cohort studies. Carcinogenesis 2014;35:25661 .

10. Zhong S, Chen W, Yu X, Chen Z, Hu Q, Zhao J. Coffee consumption and risk of prostate cancer: an up-to-date meta-analysis. Eur J Clin Nutr 2014;68:330-7.

11. Huang TB, Guo ZF, Zhang XL, Zhang XP, Liu H, Geng $\mathrm{J}$, et al. Coffee consumption and urologic cancer risk: a meta-analysis of cohort studies. Int Urol Nephrol 2014;46: 1481-93.

12. Liu H, Hu GH, Wang XC, Huang TB, Xu L, Lai P, et al. Coffee consumption and prostate cancer risk: a metaanalysis of cohort studies. Nutr Cancer 2015;67:392-400.

13. Wang A, Wang S, Zhu C, Huang H, Wu L, Wan X, et al. Coffee and cancer risk: a meta-analysis of prospective observational studies. Sci Rep 2016;6:33711.

14. Bae JM. Meta-epidemiology. Epidemiol Health 2014;36: e2014019.

15. Severson RK, Nomura AM, Grove JS, Stemmermann GN. 
A prospective study of demographics, diet, and prostate cancer among men of Japanese ancestry in Hawaii. Cancer Res 1989;49:1857-60.

16. Le Marchand L, Kolonel LN, Wilkens LR, Myers BC, Hirohata T. Animal fat consumption and prostate cancer: a prospective study in Hawaii. Epidemiology 1994;5:27682.

17. Allen NE, Sauvaget C, Roddam AW, Appleby P, Nagano J, Suzuki G, et al. A prospective study of diet and prostate cancer in Japanese men. Cancer Causes Control 2004;15: 911-20.

18. Nilsson LM, Johansson I, Lenner P, Lindahl B, Van Guelpen B. Consumption of filtered and boiled coffee and the risk of incident cancer: a prospective cohort study. Cancer Causes Control 2010;21:1533-44.

19. Wilson KM, Kasperzyk JL, Rider JR, Kenfield S, van Dam RM, Stampfer MJ, et al. Coffee consumption and prostate cancer risk and progression in the Health Professionals Follow-up Study. J Natl Cancer Inst 2011; 103:876-84.

20. Shafique K, McLoone P, Qureshi K, Leung H, Hart C, Morrison DS. Coffee consumption and prostate cancer risk: further evidence for inverse relationship. Nutr J 2012; 11:42.

21. Bosire C, Stampfer MJ, Subar AF, Wilson KM, Park Y, Sinha R. Coffee consumption and the risk of overall and fatal prostate cancer in the NIH-AARP Diet and Health Study. Cancer Causes Control 2013;24:1527-34.

22. Discacciati A, Orsini N, Andersson SO, Andrén O, Johansson JE, Mantzoros CS, et al. Coffee consumption and risk of localized, advanced and fatal prostate cancer: a population-based prospective study. Ann Oncol 2013;24:
1912-8.

23. Li Q, Kakizaki M, Sugawara Y, Tomata Y, Watanabe T, Nishino Y, et al. Coffee consumption and the risk of prostate cancer: the Ohsaki Cohort Study. Br J Cancer 2013; 108:2381-9.

24. Tverdal A. Boiled coffee consumption and the risk of prostate cancer: follow-up of 224,234 Norwegian men 2069 years. Br J Cancer 2015;112:576-79.

25. Bae JM, Kim EH. Citation discovery tools for conducting adaptive meta-analyses to update systematic reviews. J Prev Med Public Health 2016;49:129-33.

26. Orsini N, Bellocco R, Greenland S. Generalized least squares for trend estimation of summarized dose-response data. Stata J 2006;6:40-57.

27. Russnes KM, Wilson KM, Epstein MM, Kasperzyk JL, Stampfer MJ, Kenfield SA, et al. Total antioxidant intake in relation to prostate cancer incidence in the Health Professionals Follow-Up Study. Int J Cancer 2014;134: 1156-65.

28. Park SY, Freedman ND, Haiman CA, Le Marchand L, Wilkens LR, Setiawan VW. Prospective study of coffee consumption and cancer incidence in non-white populations. Cancer Epidemiol Biomarkers Prev 2018;27:928-35.

29. Sen A, Papadimitriou N, Lagiou P, Perez-Cornago A, Travis RC, Key TJ, et al. Coffee and tea consumption and risk of prostate cancer in the European Prospective Investigation into Cancer and Nutrition. Int J Cancer 2019; 144:240-50.

30. Discacciati A, Orsini N, Wolk A. Coffee consumption and risk of nonaggressive, aggressive and fatal prostate cancer--a dose-response meta-analysis. Ann Oncol 2014;25: 584-91. 